\title{
Estudio y caracterización de vidriados vitrocerámicos basados en piroxeno
}

\author{
F. LUCAS' ${ }^{1}$, A. BELDA ${ }^{1}$, F.J. TORRES ${ }^{2}$, J. ALARCÓN ${ }^{2}$ \\ 'Departamento de I+D. FRITTA S.L. Castellón, España \\ ${ }^{2}$ Departamento de Química Inorgánica. Universidad de Valencia. 46100 Burjassot (Valencia), España
}

\begin{abstract}
En este trabajo se presenta una metodología para desarrollar vidriados vitrocerámicos basados en piroxeno. En primer lugar se ha estudiado, mediante difracción de rayos X y microscopía electrónica de barrido, el efecto de diferentes aditivos en la cristalización monofásica de piroxeno a partir de vidrios en el sistema $\mathrm{CaO} \cdot \mathrm{MgO} \cdot \mathrm{Al}_{2} \mathrm{O}_{3} \cdot \mathrm{SiO}$. Posteriormente, se han determinado los intervalos de sinterización y características térmicas de los vidrios, mediante microscopía de calefacción y dilatometría. Finalmente, con los vidrios seleccionados se han preparado piezas esmaltadas mediante tratamientos térmicos utilizados habitualmente en la industria de pavimento y revestimiento, comprobando la reproducibilidad de las características microestructurales obtenidas en los estudios iniciales en vidrios. Se han medido sobre las piezas esmaltadas diferentes propiedades que permiten confirmar su potencial aplicación en procesados industriales de monococción y/o bicocción.
\end{abstract}

Palabras clave: vidriado vitrocerámico, piroxeno, microestructura, propiedades mecánicas.

\section{Study and characterization of pyroxene-based glass-ceramic glazes.}

A method was proposed to develop pyroxene-based glass-ceramic glazes. First, was studied, by X-ray diffraction and scanning electron microscopy, the effect of several additives in the monophasic crystallization of pyroxene from glasses in the $\mathrm{CaO} \cdot \mathrm{MgO} \cdot \mathrm{Al}_{2} \mathrm{O}_{3} \cdot \mathrm{SiO}_{2}$ quaternary system. After, it was determined the sinterization intervals and thermal properties of the glasses, by hot stage microscopy and dilatometry. Finally, some studied glasses were chosen and glazed tiles were developed under fast firing wall- and floor-tile industrial cycles. The results proved the reproducibility of the microstructural characteristics obtained in the previous study with glasses. The measurement of different mechanical properties confirmed their potential application in nowadays industrial processing.

Key words: glass-ceramic glaze, pyroxene, microstructure, mechanical properties

\section{INTRODUCCIÓN}

Los materiales vitrocerámicos son sólidos policristalinos obtenidos por cristalización controlada de vidrios (1-3) y poseen un conjunto de propiedades interesantes, razón por la cual se utilizan en diversas aplicaciones (4-7). Generalmente, este tipo de materiales se ha estudiado en forma monolítica, ya que en materiales particulados el efecto de la superficie de las partículas favorece la nucleación heterogénea y se dificulta el control del crecimiento cristalino.

Los vidriados utilizados en la fabricación de piezas cerámicas de pavimento y revestimiento están constituidos esencialmente por un vidrio o frita que se desarrolla durante el proceso de cocción generando una capa uniforme de material que sella los poros y permite la decoración de la pieza. La mejora de las propiedades mecánicas del vidriado se consigue mediante la adición de fases cristalinas de elevada dureza, hecho que puede afectar las características estéticas del vidriado. La generación de fases cristalinas en el vidrio, formación de un vitrocerámico, durante el proceso de cocción del vidriado puede evitar estas dificultades y al mismo tiempo mejorar las propiedades mecánicas de este tipo de materiales (8). De esta forma se pueden obtener productos de pavimento y revestimiento innovadores que la industria cerámica de nuestro entorno necesita para competir en mercados cada vez más exigentes.
Los vidrios basados en los sistemas $\mathrm{MgO} \cdot \mathrm{Al}_{2} \mathrm{O}_{3} \cdot \mathrm{SiO}_{2}$ y $\mathrm{CaO} \cdot \mathrm{MgO} \cdot \mathrm{Al}_{2} \mathrm{O}_{3} \cdot \mathrm{SiO}_{2}$ resultan interesantes para desarrollar, utilizando los aditivos adecuados (9), vidriados vitrocerámicos con propiedades interesantes debido a la cristalización de fases como cordierita, mullita o piroxeno (10-13).

El principal objetivo del presente trabajo consiste en el planteamiento de una metodología básica para el estudio de vidrios que puedan utilizarse en la formulación y posterior desarrollo de vidriados vitrocerámicos de piroxeno, en las condiciones empleadas en la producción industrial. El conocimiento de las principales características (sinterización, reactividad y microestructura) de este tipo de sistemas permitirá mejorar las propiedades del producto final.

\section{PROCEDIMIENTO EXPERIMENTAL}

\subsection{Preparación de los vidrios}

Se formuló una composición de referencia, denominada A, dentro del campo primario de cristalización de diopsido en el sistema $\mathrm{CaO} \cdot \mathrm{MgO} \cdot \mathrm{Al}_{2} \mathrm{O}_{3} \cdot \mathrm{SiO}_{2}$. A partir de ésta se preparó un conjunto de composiciones en las que se introdujeron algunos aditivos, $\mathrm{TiO}_{2}, \mathrm{~B}_{2} \mathrm{O}_{3}$ y mezclas de $\mathrm{Na}_{2} \mathrm{O}$ y $\mathrm{K}_{2} \mathrm{O}$, en cantidades 
inferiores al $4 \%$ en peso sobre $100 \mathrm{~g}$ del vidrio de referencia. En la formulación de las composiciones se mantienen constantes las relaciones molares CaO:MgO: $\mathrm{Al}_{2} \mathrm{O}_{3}: \mathrm{SiO}_{2}$. La tabla I muestra la composición y la nomenclatura empleada.

TABLA I. COMPOSICIÓN Y NOMENCLATURA DE LOS VIDRIOS PREPARADOS.

\begin{tabular}{|c|c|c|c|c|c|c|}
\hline & \multicolumn{7}{|c|}{ Composición (\% en peso) } \\
\hline Óxido & A & B & C & D & E & F \\
\hline $\mathrm{SiO}_{2}$ & 60.0 & 57.7 & 55.5 & 60.0 & 57.7 & 55.5 \\
\hline $\mathrm{Al}_{2} \mathrm{O}_{3}$ & 14.0 & 13.5 & 13.0 & 11.0 & 10.5 & 10.2 \\
\hline $\mathrm{MgO}$ & 10.0 & 9.6 & 9.3 & 10.0 & 9.6 & 9.2 \\
\hline $\mathrm{CaO}$ & 16.0 & 15.4 & 14.8 & 16.0 & 15.4 & 14.8 \\
\hline $\mathrm{TiO}_{2}$ & - & 3.8 & 3.7 & - & - & 3.7 \\
\hline $\mathrm{B}_{2} \mathrm{O}_{3}$ & - & - & - & 3.0 & 2.9 & 2.8 \\
\hline $\mathrm{K}_{2} \mathrm{O}$ & - & - & 2.8 & - & 2.9 & 2.8 \\
\hline $\mathrm{Na}_{2} \mathrm{O}$ & - & - & 0.9 & - & 1.0 & 0.9 \\
\hline
\end{tabular}

Las materias primas de calidad industrial empleadas fueron caolín tipo $\mathrm{B}$ y cuarzo SE-8 (como fuentes de $\mathrm{SiO}_{2}$ y $\mathrm{Al}_{2} \mathrm{O}_{3}$ ), dolomita $(\mathrm{CaO}$ y $\mathrm{MgO})$, magnesita $(\mathrm{MgO})$, anatasa $\left(\mathrm{TiO}_{2}\right)$, $\mathrm{BO}_{3} \mathrm{H}_{3}, \mathrm{NaNO}_{3}$ y $\mathrm{KNO}_{3}$.

Los vidrios de diferente composición se obtuvieron mediante fusión a $1600{ }^{\circ} \mathrm{C}$ de las cantidades adecuadas de materias primas y posterior enfriamiento en agua. Para llevar a cabo las fusiones se emplearon crisoles de alúmina y horno de fusión eléctrico. Las fritas obtenidas se molturaron hasta tamaños de partícula inferiores a $30 \mu \mathrm{m}$. A continuación, se obtuvieron pastillas de polvo de vidrio por prensado a baja presión $(\sim 5 \mathrm{MPa})$ y se trataron térmicamente a diferentes temperaturas con tiempos de retención de 2 horas.

Con las fritas objeto de estudio se esmaltaron probetas de gres porcelánico y se cocieron a diferentes temperaturas entre 1100 y $1200{ }^{\circ} \mathrm{C}$ siguiendo ciclos de cocción empleados en el procesado industrial.

\subsection{Técnicas de caracterización}

La evolución de los vidrios se estudió mediante diferentes técnicas instrumentales.

La caracterización dilatométrica se realizó en probetas de polvo de vidrio prensado (dilatómetro Bahr DIL $801 \mathrm{~L}$ ) en los intervalos $100-300{ }^{\circ} \mathrm{C}$ y $300-500{ }^{\circ} \mathrm{C}$.

La sinterización se estudió en probetas de polvo de vidrio (microscopio de calefacción Misura 3) en el intervalo de temperatura $40-1300{ }^{\circ} \mathrm{C}$ con una velocidad de calentamiento de 50 ${ }^{\circ} \mathrm{C} /$ minuto.

El análisis de las fases cristalinas generadas en los vidrios tratados térmicamente se realizó mediante difracción de polvo de rayos $X$ (difractómetro Siemens D-5000, con filtro de Ni, radiación $\mathrm{CuK}_{\alpha}$ por monocromador de grafito). Los diagramas de difracción de rayos $X$ se registraron en el intervalo $2 \theta$ de $5^{\circ}$ a $65^{\circ}$ con un paso de $0.02^{\circ}$ cada 5 segundos.

La microestructura de las muestras tratadas térmicamente se observó, en superficies de fractura atacadas con una disolución de HF para eliminar parte de fase vítrea, mediante microscopía electrónica de barrido (MEB) de emisión de campo a $30 \mathrm{kV}$ (microscopio Hitachi S-4100).

Las propiedades mecánicas de los vidriados se determinaron mediante la medida de microdureza Vickers (microdurómetro Matsuzawa MHT-1) utilizando una carga de 200 gramos durante 25 segundos, y mediante ensayos de resistencia a la abrasión a 1500 revoluciones según la norma ISO-10545-7. Además se realizaron ensayos de resistencia de los vidriados

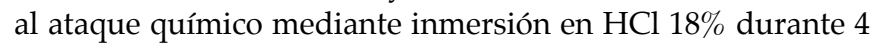
días según la norma ISO-10545-13.

\section{RESULTADOS Y DISCUSIÓN}

\subsection{Sinterización y expansión térmica de los vidrios}

En la tabla II se muestran los resultados obtenidos mediante ensayos dilatométricos. Como puede apreciarse la introducción de pequeñas cantidades de aditivos provoca cambios significativos en los coeficientes de expansión térmica de manera que es posible ajustarlos dentro de los intervalos requeridos para el correcto desarrollo de vidriados sobre soportes de gres o gres porcelánico. En la misma tabla se muestran los valores de temperatura de transición vítrea $\left(\mathrm{T}_{\mathrm{g}}\right)$ y de reblandecimiento $\left(\mathrm{T}_{\mathrm{sp}}\right)$. De los valores de $\mathrm{T}_{\mathrm{g}}$ obtenidos se deduce que en general la temperatura de transición vítrea disminuye en los vidrios con aditivos. Esta disminución es mayor en los vidrios conteniendo tanto óxidos alcalinos como $\mathrm{B}_{2} \mathrm{O}_{3}$.

TABLA II. CARACTERIZACIÓN DE LOS VIDRIOS MEDIANTE DILATOMETRÍA.

\begin{tabular}{|l|c|c|c|c|c|c|}
\hline & \multicolumn{6}{|c|}{ Composición } \\
\hline & A & B & C & D & E & F \\
\hline$\alpha \cdot 10^{-7}\left(\mathrm{~K}^{-1}\right)\left(100-300{ }^{\circ} \mathrm{C}\right)$ & 53.41 & 56.97 & 67.29 & 54.87 & 75.70 & 75.31 \\
\hline$\alpha \cdot \mathbf{1 0}^{-7}\left(\mathrm{~K}^{-1}\right)\left(300-500^{\circ} \mathrm{C}\right)$ & 61.09 & 61.49 & 75.25 & 61.34 & 80.54 & 79.47 \\
\hline $\mathrm{T}_{\mathrm{g}}\left({ }^{\circ} \mathrm{C}\right)^{*}$ & 753 & 744 & 723 & 725 & 709 & 672 \\
\hline $\mathrm{T}_{\mathrm{sp}}\left({ }^{\circ} \mathrm{C}\right)^{+}$ & 814 & 805 & 845 & 792 & 990 & 990 \\
\hline
\end{tabular}

${ }^{ } T_{g}=$ temperatura de transición vitrea $;{ }^{\dagger} T_{s p}=$ temperatura de reblandecimiento

Para comprobar la influencia de la composición del vidrio en el proceso de sinterización se realizaron ensayos de microscopía de calefacción en los vidrios de las diferentes composiciones. En la figura 1 se muestra la variación de la altura de la probeta en función de la temperatura. El análisis de las curvas de sinterización demuestra que los vidrios que contienen óxidos de elementos alcalinos y alcalino-térreos en su composición, vidrios $\mathrm{C}$, E y F, el proceso de sinterización practicamente finaliza a temperaturas inferiores (aproximadamente a $900{ }^{\circ} \mathrm{C}$ ) que en el resto de composiciones.

\subsection{Cristalización de los vidrios de partida}

Para determinar la influencia de los diversos aditivos y combinaciones de estos en la cristalización de piroxeno como fase única se calcinaron, en horno de laboratorio, los vidrios en forma de polvo prensado, a baja presión, a 1000 y $1100{ }^{\circ} \mathrm{C}$ con 2 horas de retención. Se seleccionaron estas temperaturas por su proximidad a la máxima temperatura alcanzada en ciclos industriales estándar de cocción de gres porcelánico. Además, las muestras se retuvieron durante 2 horas a dichas temperaturas para aproximar el sistema al equilibrio.

La evolución de las fases cristalinas que se generan en el proceso de desvitrificación es similar para las dos temperaturas ensayadas. En la figura 2 se muestran los difractogramas de los vidrios calcinados a $1100{ }^{\circ} \mathrm{C}$. Estos resultados ponen de manifiesto que la composición A, que sólo contiene los óxidos del sistema cuaternario $\mathrm{CaO} \cdot \mathrm{MgO} \cdot \mathrm{Al}_{2} \mathrm{O}_{3} \cdot \mathrm{SiO}_{2^{\prime}}$, presenta 


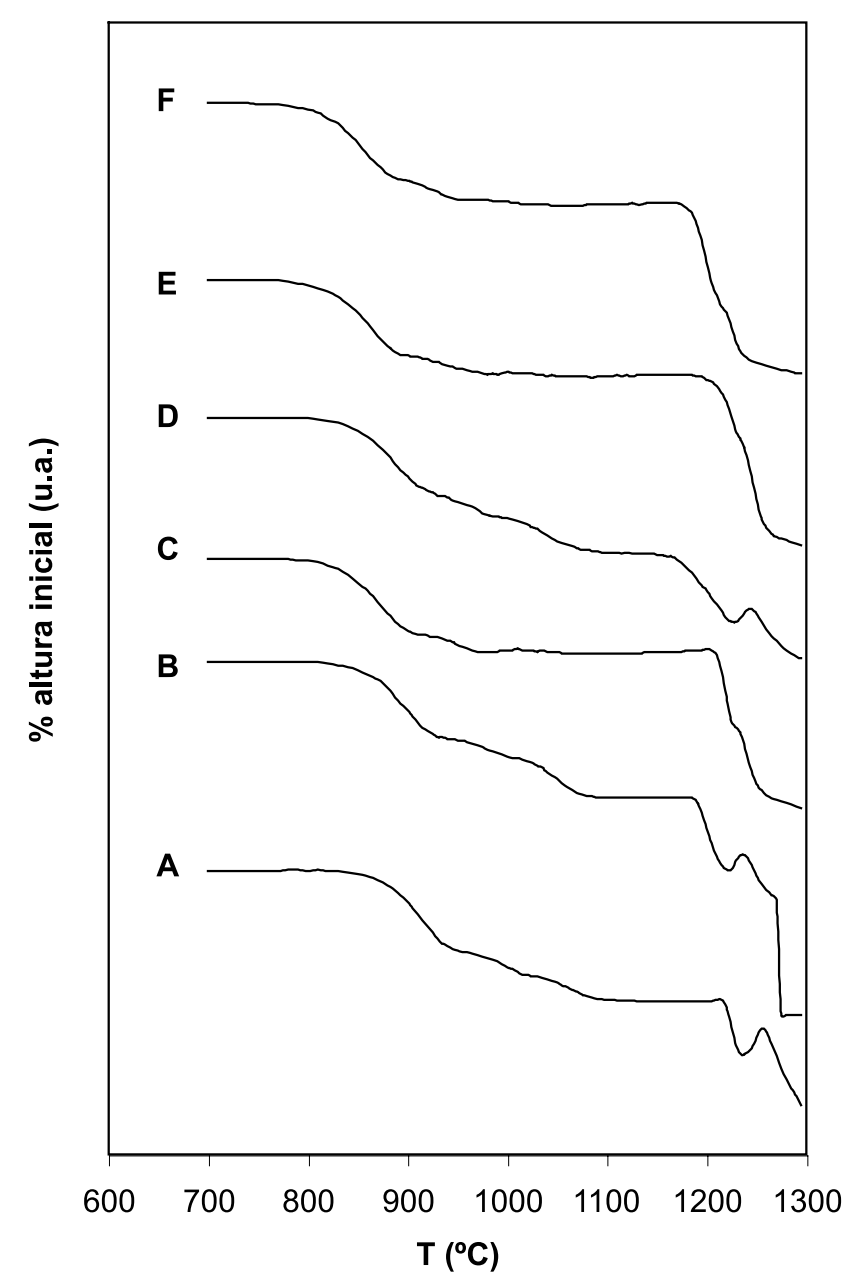

Figura 1. Curvas de sinterización de vidrios de diferente composición en función de la temperatura.

anortita como fase mayoritaria y piroxeno y cristobalita como minoritarias. Las composiciones que contienen $\mathrm{TiO}_{2} \mathrm{o}_{\mathrm{B}_{2}} \mathrm{O}_{3^{\prime}}$ denominadas $\mathrm{B}$ y $\mathrm{D}$ respectivamente, presentan anortita y piroxeno como fases mayoritarias y cristobalita como minoritaria. Por otro lado, las composiciones que contienen los aditivos anteriores junto con una mezcla de $\mathrm{Na}_{2} \mathrm{O}$ y $\mathrm{K}_{2} \mathrm{O}$, denominadas $\mathrm{C}$ y $\mathrm{E}$ respectivamente, desvitrifican piroxeno como fase mayoritaria y anortita como minoritaria. El difractograma de la muestra correspondiente a la composición que contiene todo el conjunto de aditivos, denominada $\mathrm{F}$, presenta piroxeno como única fase cristalina junto a la fase vítrea residual.

Los resultados anteriores constatan la necesidad de utilizar diversos aditivos para cristalizar piroxeno como fase única. El $\mathrm{TiO}_{2}$ favorece la generación de núcleos a partir de los cuales tiene lugar el crecimiento de los cristales (14). La presencia de $\mathrm{B}_{2} \mathrm{O}_{3}$ se justifica por el papel de este aditivo, concretamente favoreciendo el proceso de sinterización (15). Además, la presencia de óxidos alcalinos y alcalino-térreos en la composición del vidrio juega un papel doble, por un lado permiten rebajar la temperatura de fusión de los vidrios y por otro favorecen la cristalización de piroxeno. Ambos hechos están relacionados con su capacidad para actuar como mineralizadores y con la potencial entrada de los cationes $\mathrm{Na}^{+}$y $\mathrm{K}^{+}$en la estructura de piroxeno (16).

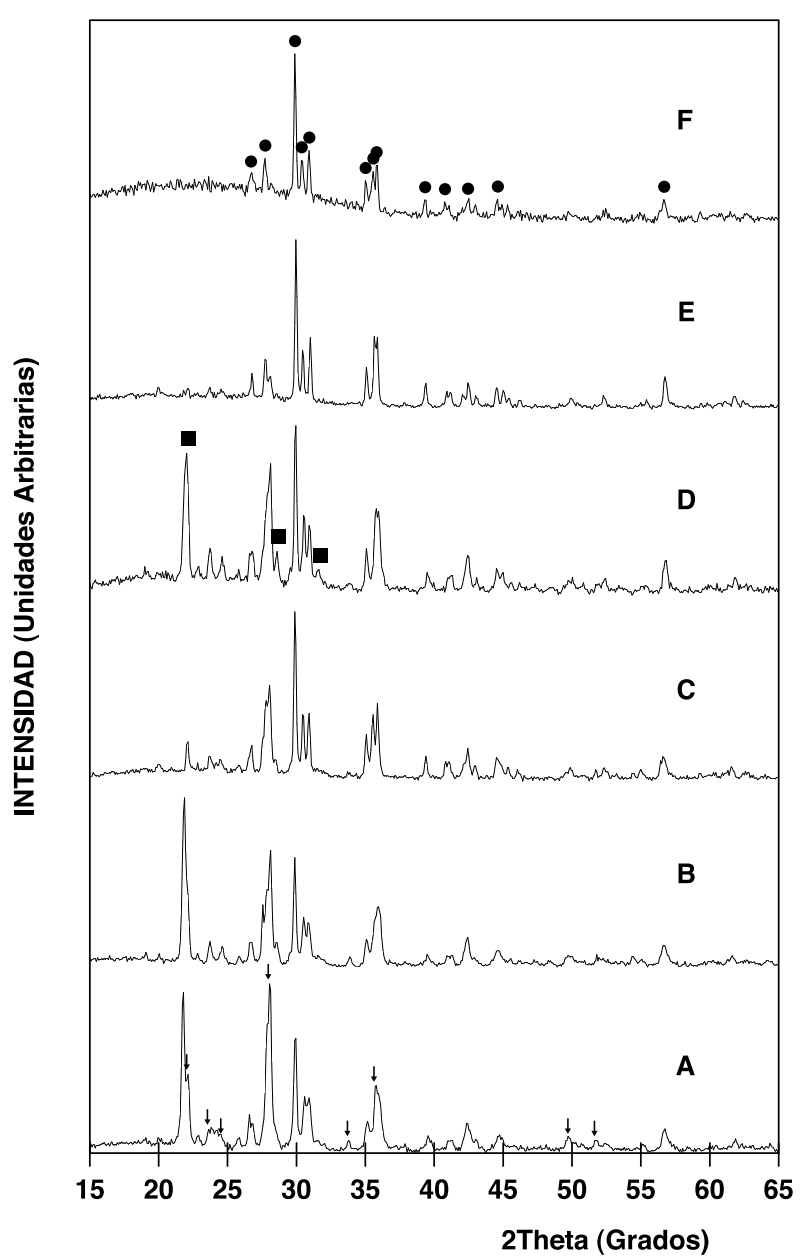

Figura 2. Difractogramas de vidrios tratados a $1100{ }^{\circ} \mathrm{C}$ durante 2 horas ( piroxeno, $\downarrow$ anortita y cristobalita).

Para el desarrollo de materiales vitrocerámicos es interesante que el proceso de sinterización y el de cristalización ocurran en intervalos de temperatura diferenciados. Los difractogramas de las muestras tratadas térmicamente mediante un programa de calentamiento similar al utilizado en el estudio de la sinterización, es decir calentando hasta $900{ }^{\circ} \mathrm{C}$ sin retención y con una velocidad de calentamiento de $50{ }^{\circ} \mathrm{C} / \mathrm{min}$, confirman que en estas condiciones las muestras son amorfas y por tanto la cristalización no se ha iniciado. En este sentido, los resultados anteriores indican que en los vidrios C, E y F la cristalización comienza cuando el proceso de sinterización del material casi ha concluido.

\subsection{Cristalización y microestructura de soportes esmaltados}

Con el fin de comprobar el efecto del tratamiento térmico en la cristalización de piroxeno se realizaron pruebas de esmaltado, de soportes de gres porcelánico, con cada una de las fritas preparadas. Las probetas se cocieron a 1155 y $1190{ }^{\circ} \mathrm{C}$ como temperatura máxima según ciclos estándar de monococción y bicocción. En las figuras 3 y 4 se muestran los difractogramas de la superficie de las piezas esmaltadas. El análisis por difracción de rayos $\mathrm{X}$ de las probetas cocidas demuestra 


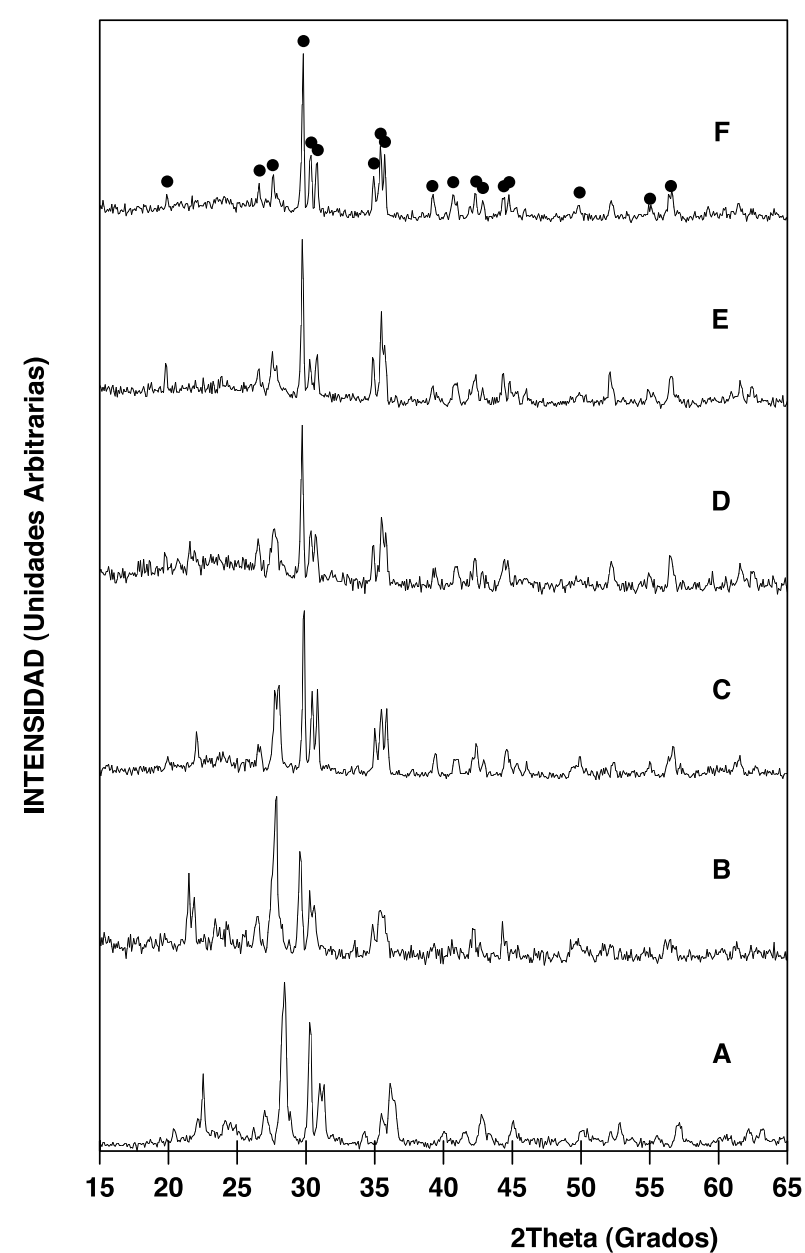

Figura 3. Difractogramas de vidriados de diferente composición calcinados según ciclo de monococción de porcelánico a $1155^{\circ} \mathrm{C}$ piroxeno).

un comportamiento en la desvitrificación similar al obtenido en ensayos de laboratorio.

La observación, mediante MEB, de la sección transversal de las piezas permite afirmar que durante el tratamiento térmico no aparecen cristales con morfología definida en el vidriado de composición A, mientras que en los vidriados con aditivos se generan cristales con morfologías definidas.

En la figura 5 se muestra la micrografía MEB correspondiente a la observación de la microestructura del vidriado de composición B cocido a $1190{ }^{\circ} \mathrm{C}$. Se observan morfologías cristalinas distintas posiblemente debido a la presencia de varias fases cristalinas tal y como se comentó anteriormente. Esto mismo se observa en los vidriados de composición C y D.

En el caso del vidriado de composición E, en el que la presencia de fases secundarias es minoritaria, se observa una microestructura (figura 6) constituida por cristales con una morfología más homogénea. La microestructura del vidriado de composición $\mathrm{F}$ (figura 7) también presenta un único tipo de cristales, distribuidos homogéneamente por toda la muestra, de tamaño inferior a $3 \mu \mathrm{m}$, con morfologías de poliedros de caras planas mejor definidos que en el resto de composiciones y que pueden asociarse sin lugar a dudas a la fase piroxeno.

Es de destacar que el aspecto estético final del vidriado es una característica que está influenciada por la temperatura

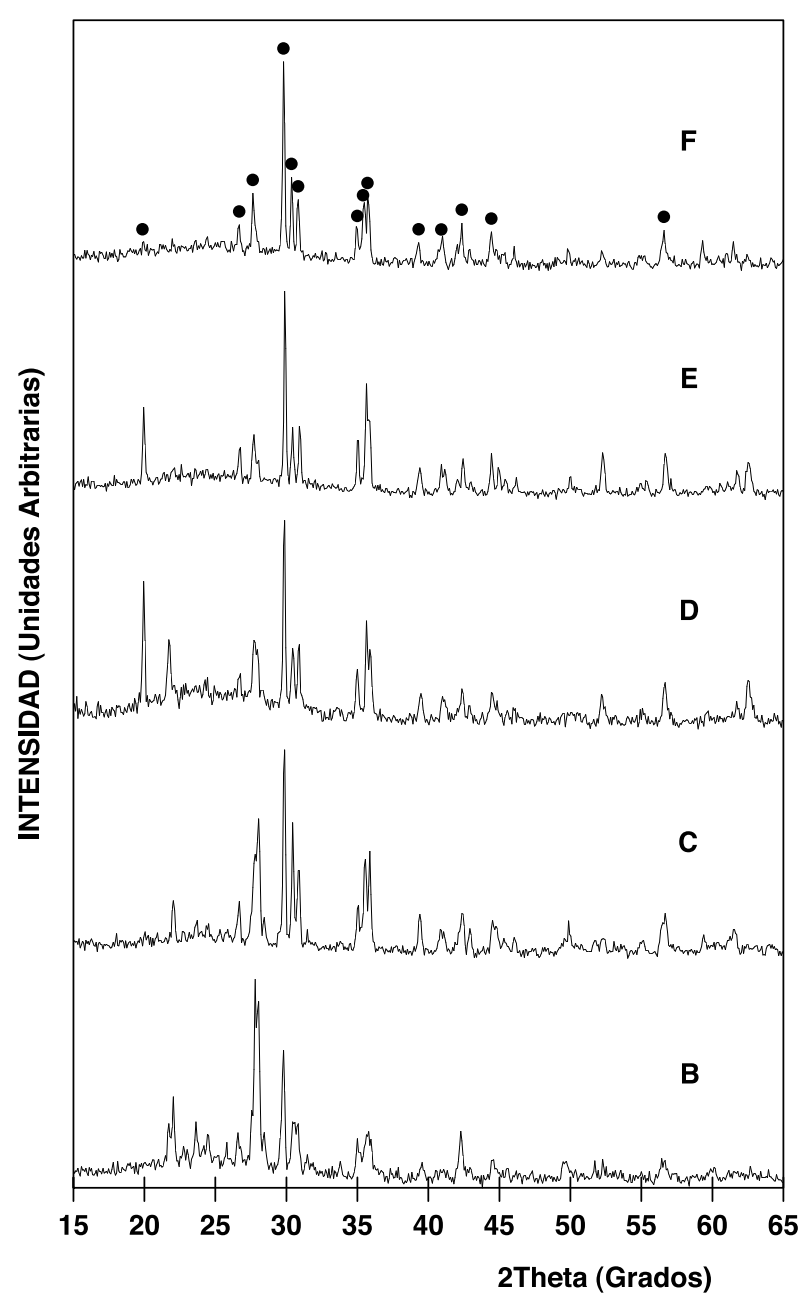

Figura 4. Difractogramas de vidriados de diferente composición calcinados según ciclo de monococción de porcelánico a $1190{ }^{\circ} \mathrm{C}$ piroxeno).

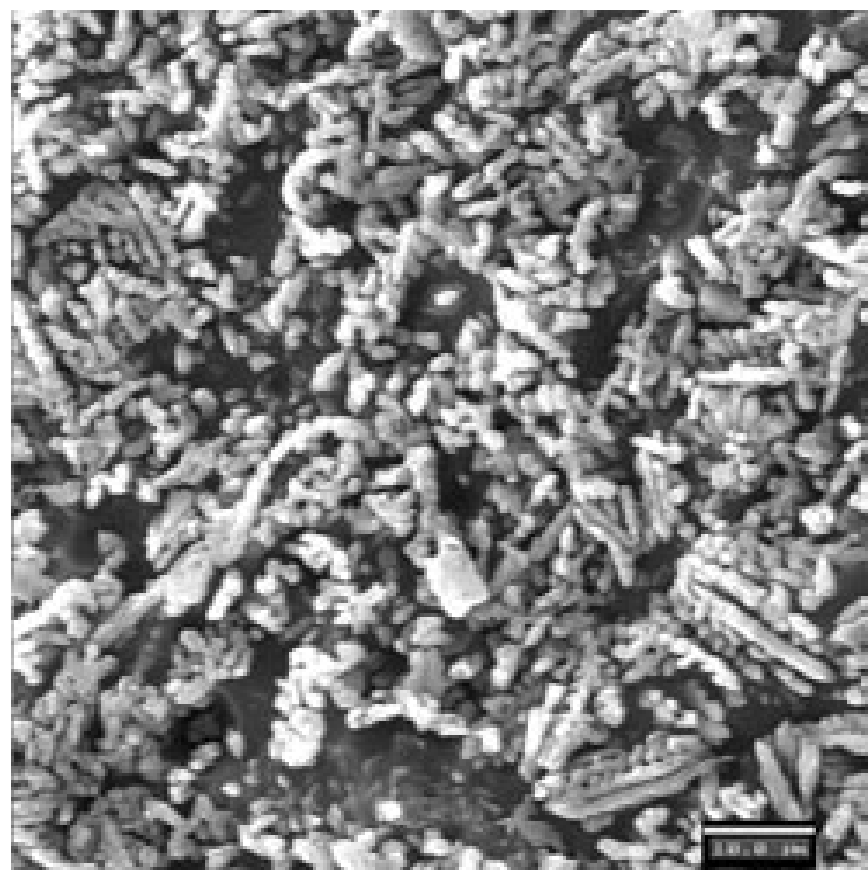

Figura 5. Micrografía de MEB del vidriado de composición B calcinado según ciclo de monococción de porcelánico a $1190{ }^{\circ} \mathrm{C}($ barra $=10 \mu \mathrm{m})$. 


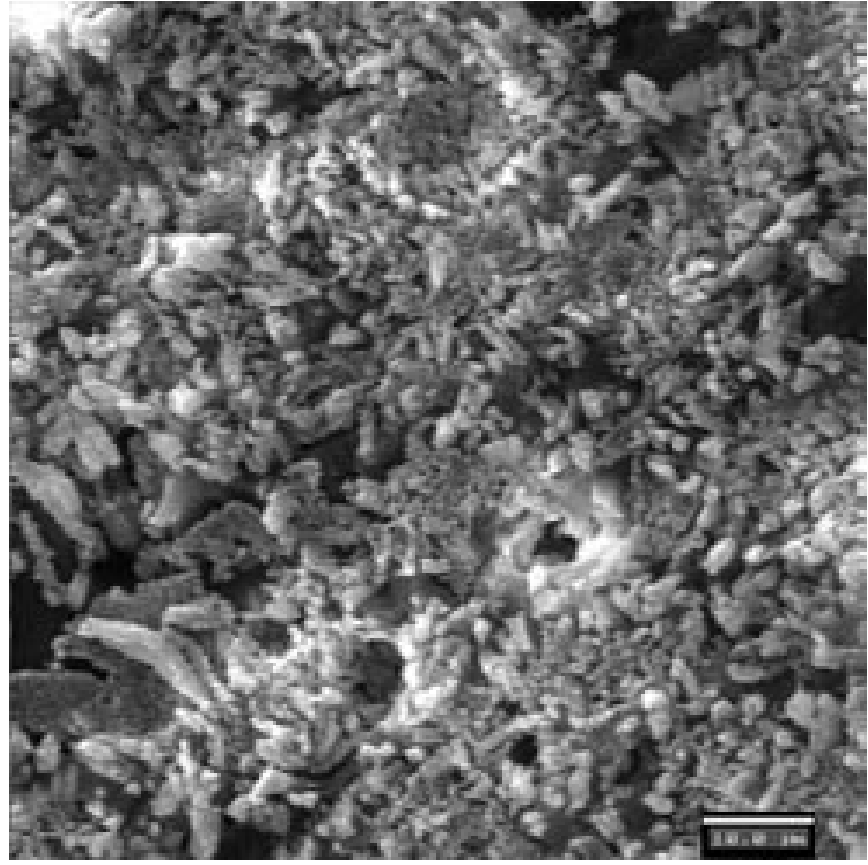

Figura 6. Micrografía de MEB del vidriado de composición E calcinado según ciclo de monococción de porcelánico a $1190^{\circ} \mathrm{C}($ barra $=10 \mu \mathrm{m})$.

máxima empleada durante el tratamiento térmico. Se observa que las probetas tratadas a $1190{ }^{\circ} \mathrm{C}$ presentan menos defectos debido a un mejor desarrollo del vidriado. En algún caso es necesario tratar térmicamente el soporte antes de esmaltar, con el fin de facilitar la desgasificación y conseguir un aspecto estético adecuado.

\subsection{Propiedades de los vidriados vitrocerámicos}

El crecimiento de cristales de piroxeno en el seno de una matriz vítrea provoca una mejora de algunas de las propiedades, principalmente mecánicas y químicas, respecto a los vidriados convencionales. Por esta razón, en los vidriados que no presentaban ningún defecto se realizaron medidas de microdureza Vickers, resistencia a la abrasión y resistencia al ataque químico. Para evaluar la resistencia a las manchas y porosidad cerrada se ha aplicado sobre la zona abrasionada carbón activo, este ensayo no ha afectado a ninguna de las composiciones ensayadas. Los resultados se resumen en la tabla III. El análisis de los resultados pone de manifiesto que el desarrollo de un vidriado vitrocerámico en el que cristaliza piroxeno provoca una mejora general de los parámetros evaluados respecto a un vidriado convencional.

TABLA III. MEDIDA DE PROPIEDADES MECÁNICAS Y QUÍMICAS DE LOS VIDRIADOS VITROCERÁMICOS.

\begin{tabular}{|l|c|c|c|}
\hline \multicolumn{1}{|c|}{ Propiedad } & $\begin{array}{c}\text { Vitrocerámico } \\
\text { B }\end{array}$ & $\begin{array}{c}\text { Vitrocerámico } \\
\text { D }\end{array}$ & $\begin{array}{c}\text { Vitrocerámico } \\
\mathbf{F}^{*}\end{array}$ \\
\hline Microdureza Vickers $\left(\mathrm{kg} / \mathrm{mm}^{2}\right)$ & $680 \pm 40$ & $680 \pm 30$ & $660 \pm 30$ \\
\hline Resistencia a la abrasión & $\begin{array}{c}\text { Sin efecto } \\
\text { visible }\end{array}$ & $\begin{array}{c}\text { Ligeramente } \\
\text { mateado }\end{array}$ & $\begin{array}{c}\text { Ligeramente } \\
\text { mateado }\end{array}$ \\
\hline Resistencia al ataque químico & Ligero ataque & Ligero ataque & Sin efecto \\
\hline
\end{tabular}

PROCESADO MEDIANTE CICLO DE BICOCCIÓN

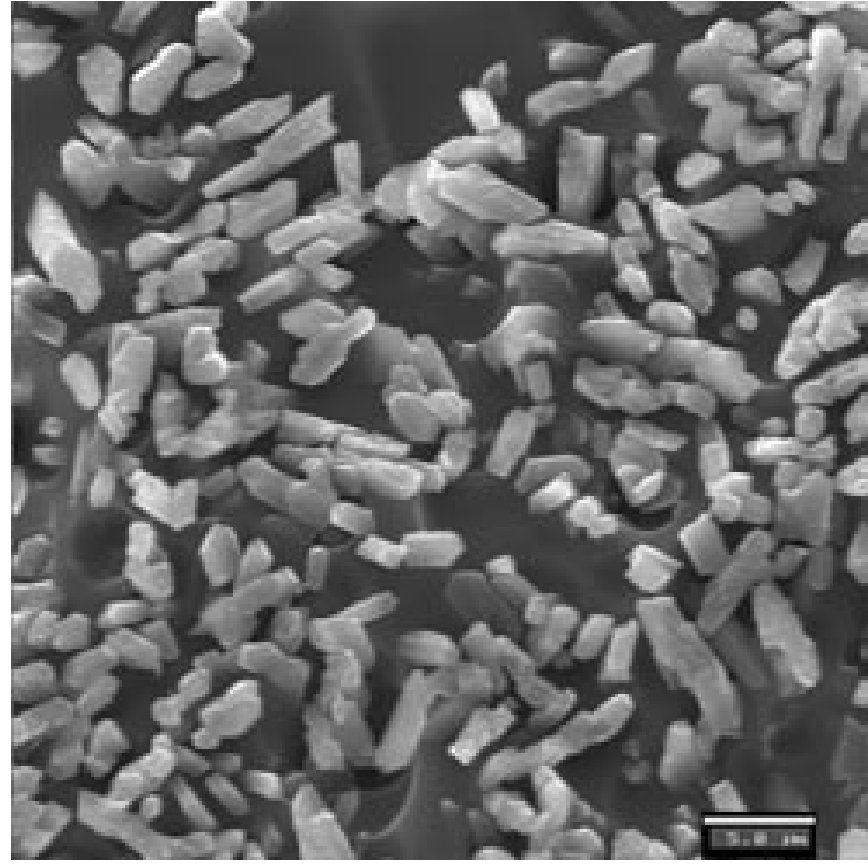

Figura 7. Micrografía de MEB del vidriado de composición F calcinado según ciclo de bicocción de porcelánico a $1190^{\circ} \mathrm{C}$ (barra $\left.=5 \mu \mathrm{m}\right)$.

\section{CONCLUSIONES}

Se ha estudiado la cristalización de vidrios formulados dentro del sistema de composición $\mathrm{CaO} \cdot \mathrm{MgO} \cdot \mathrm{Al}_{2} \mathrm{O}_{3} \cdot \mathrm{SiO}_{2}$ y se ha comprobado que para obtener materiales vitrocerámicos monofásicos de piroxeno es necesario introducir en la composición pequeñas cantidades de aditivos tales como $\mathrm{TiO}_{2}, \mathrm{~B}_{2} \mathrm{O}_{3}$ y mezclas de $\mathrm{Na}_{2} \mathrm{O}$ y $\mathrm{K}_{2} \mathrm{O}$.

Los estudios sobre soportes esmaltados y tratados en condiciones industriales revelan la generación de cristales de piroxeno cuya morfología depende de la combinación de aditivos empleada. La temperatura de cocción influye principalmente en el correcto desarrollo de la capa superficial del vidriado. Se ha comprobado que este tipo de vidriados presentan una mejora en las propiedades mecánicas y químicas frente a los vidriados convencionales.

\section{AGRADECIMIENTOS}

Este trabajo ha sido financiado por el Centro para el Desarrollo Tecnológico Industrial (CDTI- Proyecto 010330) y por el Programa de Fomento para la Investigación Técnica (PROFITProyecto FIT-033333-2001-238).

\section{BIBLIOGRAFÍA}

1. P. W. Mc Millan. “Glass Ceramics”, $2^{\text {nd }}$ Ed., Academic Press, London (1979). 2. Z. Strand. "Glass Ceramic Materials", Vol.8, Glass Science and Technology, Elsevier, Amsterdam (1986).

3. J. Ma. Rincón. “Principles of Nucleation and Controlled Crystallization of Glasses". Polym.-Plast. Technol. Eng., 31 [3-4] 309-357 (1992)

4. R. R. Tumala. "Ceramic and Glass-Ceramic Packaging in the 1990s". J. Am. Ceram. Soc., 74 [5], 895-908 (1991).

5. S. H. Knickerbocker, A. H. Kumar, L. W. Herron. “Cordierite Glass-Cera- 
mics for Multilayer Ceramic Packaging". Am. Ceram. Soc. Bull., 72 [1] 90-95 (1993).

6. G. H. Beall, L. R. Pinckney. "Nanophase Glass-Ceramics". J. Am. Ceram. Soc., 82 [1], 5-16 (1999).

7. I. Vicente-Mingarro, P. Callejas, J. Ma. Rincón. “Materiales Vitrocerámicos: El Proceso Vitrocerámico". Bol. Soc. Esp. Ceram. V., 32 [3] 157-167 (1993).

8. Nota técnica del Editor."CER-GLASS: Desarrollo de Vidriados Vitrocerámicos para Pavimentos de Gres con Alta Resistencia a la Abrasión y Alto Brillo". Bol. Soc. Esp. Cerám. V., 33 [2], 99-102 (1994).

9. F. J. Torres, J. Alarcón. "Effect of Additives on the Crystallization of Cordierite-based Glass-Ceramics as Glazes for Floor Tiles". J. Eur. Ceram. Soc., 23, 817-826 (2003)

10. A. M. Ferrari, L. Barbieri, C. Leonelli, T. Manfredini, C. Siligardi, A. B. Corradi. "Feasibility of Using Cordierite Glass-Ceramics as Tile Glazes". J. Am. Ceram. Soc., 80 [7], 1757-1766 (1997).

11. F. J. Torres, J. Alarcón. “Estudio de la cristalización de mullita en vidrios del sistema CMAS". Comunicación interna.
12. H. S. Kim, R. D. Rawling, P. S. Rogers. "Sintering and Crystallization Phenomena in Silceram Glass", J. Mater. Sci., 23, 2622-2630 (1988).

13. J. Ma. Rincón, M. Romero, J. Marco, V. Caballer. "Some aspects of crystallization microstructure on new glass-ceramics glazes". Mat. Res. Bull., 33 [8] 1159-1164 (1998)

14. G. Baldi, E. Generalli, C. Leonelli, T. Manfredini, G. C. Pellacani, C. Siligardi. "Effects of nucleating agents on diopside crystallization in new glass-ceramics for tile-glaze application", J. Mater. Sci., 30, 3251-3255 (1995).

15. Y. Sung. "The effect of additives on the crystallization and sintering of $2 \mathrm{MgO}-2 \mathrm{Al}_{2} \mathrm{O}_{3}-5 \mathrm{SiO}_{2}{ }^{\prime \prime}$, J. Mater. Sci., 31, 5421-5427 (1996)

16. M. Cameron, J. J. Papike. "Structural and chemical variations in pyroxenes", Am. Mineral., 66, 1-50, (1981).

Recibido: 01.12 .03

Aceptado: 25.05.04 